\title{
Extending the in vivo Half-Life of Adalimumab Fab via Sortase A-Mediated Conjugation of Adalimumab Fab with Modified Fatty Acids
}

\author{
Qing-Bin Zhang ${ }^{1}$ Si-Da Ruan ${ }^{1} \quad$ Yong Wu ${ }^{1,2}$ Jin-Hua \\ 1 State Key Laboratory of New Drug and Pharmaceutical Process, \\ Shanghai Institute of Pharmaceutical Industry, China State Institute \\ of Pharmaceutical Industry, Shanghai, People's Republic of China \\ 2 Shanghai Duomirui Bio-Technology Co., Ltd., Shanghai, People's \\ Republic of China \\ ${ }^{3}$ School of Pharmacy, Fudan University, Shanghai, People's Republic \\ of China
}

Pharmaceutical Fronts 2020;2:e160-e167.

\begin{abstract}
Address for correspondence Jun Feng, PhD, State Key Laboratory of New Drug and Pharmaceutical Process, Shanghai Institute of Pharmaceutical Industry, 285 Gebaini Road, Shanghai 201203, People's Republic of China (e-mail: fengj31@aliyun.com).
\end{abstract}

\begin{abstract}
Keywords

- TNF- $\alpha$

- adalimumab Fab

- half-life

- FA motifs

- sortase A

Adalimumab, a full-length monoclonal antibody, is widely used as an anti-tumor necrosis factor- $\alpha$ (anti-TNF- $\alpha$ ) agent. In this article, we aimed to prolong the in vivo half-life of adalimumab antigen-binding fragment (Fab) through Sortase A (SrtA)mediated conjugation of its Fab with fatty acid (FA). In our study, adalimumab Fab analog was prepared by adding an SrtA recognition sequence (LPETGG) and $\mathrm{His}_{6}$ tag to the heavy chain $\mathrm{C}$-terminal of the Fab via $\left(\mathrm{G}_{4} \mathrm{~S}\right)_{3}$ linker. Four FA motifs with different linkers were designed and synthesized by solid-phase methodology, then conjugated with the Fab analog using SrtA to produce Fab bioconjugates. The successful generation of four Fab bioconjugates (Fab-FA1, Fab-FA2, Fab-FA3, and Fab-FA4) was confirmed by SDS-PAGE (sodium dodecyl sulfate polyacrylamide gel electrophoresis) and mass spectrometry. Then, the bioactivities and half-life of these Fab bioconjugates were examined using TNF- $\alpha$-/human serum albumin (HSA)-binding enzyme-linked immunosorbent assay, cytotoxicity assay, and pharmacokinetic study, respectively. All Fab bioconjugates exhibited similar TNF- $\alpha$-neutralizing activities when compared with the Fab analog, even in the presence of albumin, indicating that there were no apparent influences on the functional site of Fab after FA modification. However, different degrees of affinity for HSA were observed among these Fab-FA bioconjugates, with Fab-FA3 exhibiting the maximal affinity. An in vivo study in mice further revealed remarkably improved pharmacokinetics of Fab- FA3 with a 15.2-fold longer plasma half-life (19.86 hours) compared with that of the Fab analog (1.31 hours). In summary, we have developed a novel long-acting adalimumab Fab bioconjugate, FabFA3, with more sustained in vivo activity, which can be used for drug development targeting TNF- $\alpha$-mediated inflammatory diseases.
\end{abstract}

received

February 2, 2021

accepted

February 16, 2021

published online

May 10, 2021
DOI https://doi.org/

$10.1055 / \mathrm{s}-0041-1728817$.

ISSN 2628-5088.
(C) 2021. The Author(s).

This is an open access article published by Thieme under the terms of the Creative Commons Attribution License, permitting unrestricted use, distribution, and reproduction so long as the original work is properly cited. (https://creativecommons.org/licenses/by/4.0/)

Georg Thieme Verlag KG, Rüdigerstraße 14, 70469 Stuttgart, Germany 


\section{Introduction}

Tumor necrosis factor- $\alpha$ (TNF- $\alpha$ ) plays an important role in the regulation of immune responses and inflammation. Thus, therapeutic strategy targeting TNF- $\alpha$ becomes promising and urgent in treating many diseases. ${ }^{1}$ Currently, several anti-TNF- $\alpha$ agents, including adalimumab, golimumab, certolizumab, infliximab, and etanercept, have been proven to be beneficial in clinical settings. ${ }^{2}$ Evidence shows that most of these TNF- $\alpha$ inhibitors are full-length monoclonal antibodies, of which adalimumab is a representative inhibitor and has been the top-selling drug for several consecutive years. ${ }^{3}$ However, as full-length monoclonal antibodies, these TNF- $\alpha$ inhibitors, including adalimumab, have been limited in clinical use due to the poor tissue penetration caused by large molecular weight as well as the high cost for production and use in the healthcare. ${ }^{4,5}$

Antigen-binding fragment (Fab) of an antibody has many advantages, such as low molecular weight, satisfactory antigen-binding capacity, facile prokaryotic expression, superior tissue penetration, and has been widely studied in the field of monoclonal antibodies. ${ }^{6}$ Fab is a preferable alternative to full-length monoclonal antibody in research and therapeutic applications. However, Fab is easily filtered through the glomerulus because of its small molecular weight, resulting in a relatively short half-life (only 16-20 hours) in vivo. ${ }^{5,7}$ To promote the clinical application of Fab, diverse strategies regarding sprolongation of the halflife of Fab have been developed, such as PEGylation, ${ }^{8}$ PASylation, ${ }^{9}$ albumin-binding peptide fusion, ${ }^{10}$ and glycine-rich homoamino-acid polymer (HAP) fusion, ${ }^{11}$ by increasing the hydrodynamic radius or specific binding to endogenous albumin.

Fatty acid (FA) is a reversible albumin-binding ligand. Conjugating FA with peptides and proteins will increase the half-life of targets. ${ }^{12}$ Reports have demonstrated that the half-life of several therapeutic biodrugs, such as semaglutide, liraglutide, insulin degludec, and insulin detemir, can be extended via their conjugation with FAs, which led to the significant enhancement in therapeutic efficiencies and the subsequent clinical application of those drugs. ${ }^{13,14}$ However, whether the half-life of Fab will be prolonged by the conjugation of Fab with FA remained unknown.

Sortase A (SrtA), a transpeptidase produced by grampositive bacteria, has been widely utilized as a ligase for covalent protein modification. ${ }^{15-17}$ In this study, we used SrtA for FA modification to prolong the half-life of Fab derived from adalimumab. The modified FAs were conjugated with Fab through SrtA mediation to generate Fab bioconjugates (Fab-FA1, Fab-FA2, Fab-FA3, and Fab-FA4). Our data showed that Fab-FA3 exhibited the most potent albumin-binding activity among Fab bioconjugates and displayed similar anti-TNF- $\alpha$ activity compared with control Fab. Moreover, pharmacokinetics of Fab-FA3 were significantly improved with a 15.2-fold longer half-life than control Fab. Fab-FA3 thus represents an ideal alternative for adalimumab in treating TNF- $\alpha$-mediated inflammatory disorders.

\section{Materials and Methods}

\section{Construction, Expression, and Purification of the Adalimumab Fab Analog}

The $\left(\mathrm{G}_{4} \mathrm{~S}\right)_{3}$ linker, SrtA recognition sequence (LPETGG), and $\mathrm{His}_{6}$ tag were introduced to the C-terminal of the heavy chain in the adalimumab Fab, generating an adalimumab Fab analog (-Fig. 1). The expression plasmids for Fab analog production in Escherichia coli (E. coli) was constructed according to a reported literature. ${ }^{18}$ Then, the adalimumab Fab analog was expressed and assembled in the periplasmic space of engineered strains under high-density fermentation, according to the reported methods. ${ }^{19,20}$

After fermentation, the culture medium of the engineered E. coli strains was centrifuged at $10,000 \times \mathrm{g}$ for 10 minutes at room temperature (r.t.). The microbial pellet was resuspended in five volumes of the suspension buffer $(60 \mathrm{mmol} / \mathrm{L}$ citric acid and $50 \mathrm{mmol} / \mathrm{L} \mathrm{MgSO}_{4}, \mathrm{pH}=4.0$ ). The bacterial suspension was homogenized three times at a low pressure (550 bar). The lysate was centrifuged at $10,000 \times \mathrm{g}$ for 10 minutes at $4^{\circ} \mathrm{C}$. The supernatant, named periplasmic fraction, was harvested and adjusted to $\mathrm{pH}=7.2$ for further purification.

Adalimumab Fab analog was purified using affinity chromatography with an AKTA Explorer 100 system (General Electric Company, United States). Briefly, the periplasmic fraction was filtrated with a $0.22-\mu \mathrm{m}$ filter and then loaded onto a $5 \mathrm{~mL}$ protein L affinity column (General Electric Company, United States) equilibrated with phosphate buffered saline (PBS). The column was rinsed with PBS followed by $0.1 \mathrm{~mol} / \mathrm{L}$ glycine- $\mathrm{HCl}$ buffer $(\mathrm{pH}=2.7)$ to harvest Fab analog proteins. The elution fractions were pooled, immediately adjusted to $\mathrm{pH} 7.2$ by addition of $1 \mathrm{~mol} / \mathrm{L}$ Tris-base solution and then buffer-exchanged to PBS. The concentration of Fab analog was determined by using a BCA Protein Assay Kit (C503021, Sangon Biotech). SDS-PAGE (sodium

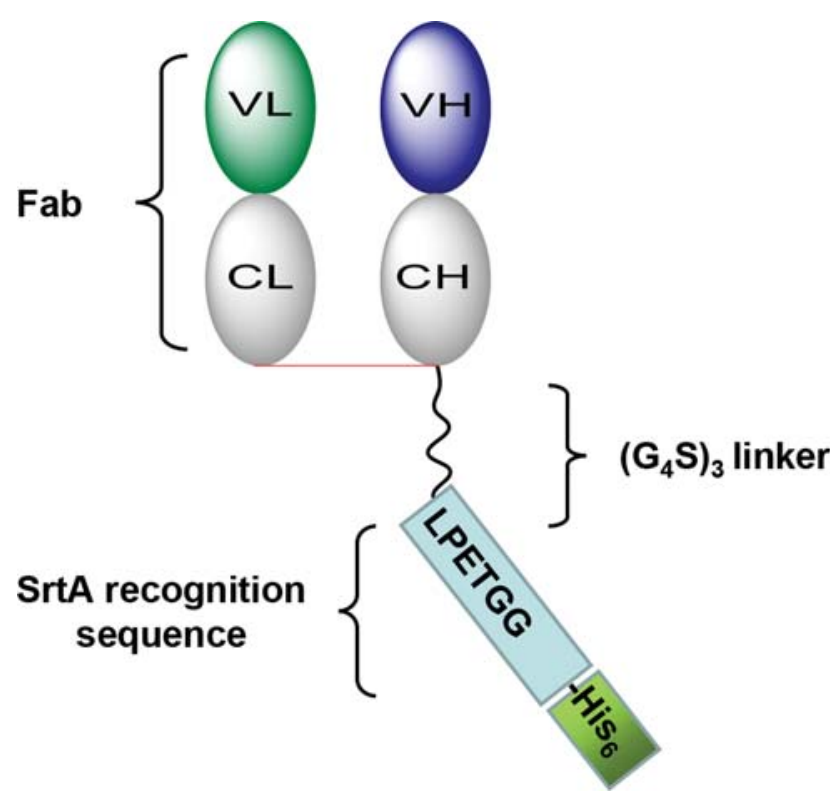

Fig. 1 Schematic representation of the Fab analog. 
dodecyl sulfate polyacrylamide gel electrophoresis) was conducted using $12 \%$ polyacrylamide to evaluate protein purity, and to assess the formation of interchain disulfide bond. The polyacrylamide gel was stained with Coomassie brilliant blue R250 (Sigma-Aldrich, United States) and then decolorized with $10 \%(v / v)$ acetic acid solution until the protein bands were visualized.

\section{Design, Synthesis, and Preparation of FA Motifs}

To facilitate site-specific modification by SrtA, we designed FA motifs containing the -Gly-Gly-Gly (-GGG) sequence at the N-terminus and -Lys (-K) at the C-terminus. As shown in -Fig. 2, different types of linkers, including 8-amino-3,6dioxaoctanoic acid (AEEA), $\mathrm{A}(\mathrm{PA})_{6}$, and $\mathrm{S}(\mathrm{PGS})_{4}$, were introduced as spacers. AEEA (a flexible hydrophilic linker) has advantages to more easily connect the functional domains, allowing for the mobility of the joined domains, which has been used in the preparation of lipid moieties in acylating position Lys26 of semaglutide. ${ }^{21} \mathrm{~A}(\mathrm{PA})_{6}$ exhibits a canonical rigid conformation and can be used to separate the functional domains effectively. ${ }^{22} \mathrm{~S}(\mathrm{PGS})_{4}$, invented by the BristolMyers Squibb Company (New York, United States), serves as a favorable linker in functional domain connection. ${ }^{23}$ Octadecanedioic acid (a binding ligand for albumin) was linked to the epsilon-amino group of $\mathrm{K}$ via the AEEA linker or not. Four FA motifs were designed and synthesized using a CS Bio solid-phase synthesizer (CS Biotechnology Co., Ltd, United States) according to Fmoc strategy through the following protected derivatives: Fmoc-Lys(Alloc)-OH, Fmoc-AEEA-OH, Fmoc-Glu(Otbu)-OH, Boc-Gly-Gly-Gly-OH, Fmoc-Ala-OH, Fmoc-Pro-OH, Fmoc-Ser-OH, Fmoc-Gly-OH, and octadecanedioic acid, 1-(1,1-dimethylethyl) ester. The FA motifs were de-protected and cleaved from the resin in a mixture of $5 \%$ dichloromethane and 95\% trifluoroacetamide (TFA) for 3 hours at r.t.. After filtration into cold diethyl ether, the crude compounds were precipitated. The precipitates were washed with diethyl ether then vacuum-dried.

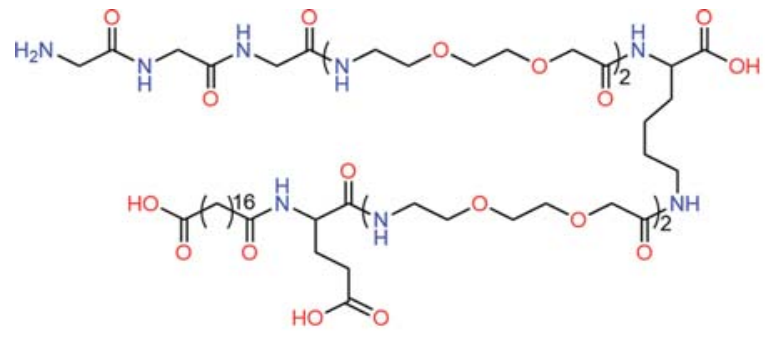

$\mathrm{NH}_{2}$-GGG-(AEEA) $)_{2}$-K( $(\varepsilon-\mathrm{NH} \text {-(AEEA) })_{2}$-E-C18-diacid)-OH (FA1)

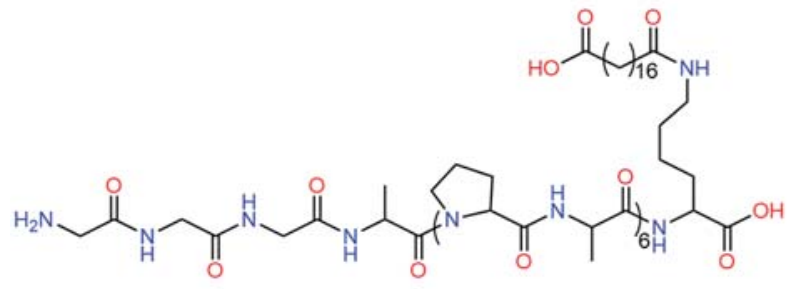

$\mathrm{NH}_{2}$-GGG-A(PA) 6 -K( $(\varepsilon-\mathrm{NH}-\mathrm{C} 18$-diacid)-OH (FA3)
The FA motifs were purified on prep-HPLC(Waters, United States) using C4-reverse-phase silica gel column chromatography. The mobile phase was set as the following: buffer $\mathrm{A}$ (0.1\% TFA $[v / v]$ water solution) and buffer B $(0.1 \%$ TFA $[v / v]$ acetonitrile solution). The target fragment was obtained by gradient elution from 20 to $37 \%$ of buffer $B$. The absorbance at $215 \mathrm{~nm}$ was monitored via ultraviolet detection. Pooled fractions containing more than 95\% pure target fragments were dried under vacuum with a rotary evaporator. The FA motifs were lyophilized and further identified via QDa electrospray ionization-mass spectrometry (Waters, United States).

\section{Preparation and Purification of Fab Bioconjugates} SrtA was prepared according to a reported study. ${ }^{24}$ Conjugation of the N-terminal of FA motif to the heavy chain Cterminus of the adalimumab Fab analog was performed as follows: $10 \mu \mathrm{mol} / \mathrm{L}$ adalimumab Fab analog, $400 \mu \mathrm{mol} / \mathrm{L}$ FA motif, $625 \mathrm{nmol} / \mathrm{L} \mathrm{SrtA}$, and $10 \mathrm{mmol} / \mathrm{L} \mathrm{CaCl}_{2}$ were mixed in a solution of $50 \mathrm{mmol} / \mathrm{L} 2$-( $\mathrm{N}$-morpholino)ethanesulfonic acid (MES) and $150 \mathrm{mmol} / \mathrm{L} \mathrm{NaCl}$ (pH 7.0). The reaction was incubated at $37^{\circ} \mathrm{C}$ for 4 hours. The product was purified using $\mathrm{Ni}^{2+}$ affinity chromatography on the AKTA Explorer 100 (General Electric Company, United States).

The reaction mixture obtained was filtrated with a $0.22-$ $\mu \mathrm{m}$ filter and loaded onto a $5 \mathrm{~mL}$ HisTrap Excel column (General Electric Company, United States) equilibrated with equilibration buffer $(20 \mathrm{mmol} / \mathrm{L} \mathrm{MES}, 150 \mathrm{mmol} / \mathrm{L}$ $\mathrm{NaCl}, \mathrm{pH}=7.4$ ). The flow-through containing the Fab bioconjugate was collected. The Fab analog and SrtA containing the $\mathrm{His}_{6}$ tag were obtained from the elution of the column using wash buffer $(20 \mathrm{mmol} / \mathrm{L}$ MES, $150 \mathrm{mmol} / \mathrm{L} \mathrm{NaCl}, 0.5$ $\mathrm{mol} / \mathrm{L}$ imidazole, $\mathrm{pH}=7.0$ ). The purity of the target molecule was analyzed via SDS-PAGE. The molecular weight was determined by UPLC-QTOF-MS (Waters, United States). The purified Fab bioconjugates were suspended in PBS and the protein concentrations were determined using a BCA kit.<smiles>[14CH3]C(=O)NCCCCC(NC(=O)COCCOCCNC(=O)CNC(=O)CNC(=O)CN)C(=O)O</smiles>

$\mathrm{NH}_{2}$-GGG-(AEEA) ${ }_{2}$-K( $\varepsilon$-NH-C18-diacid)-OH (FA2)<smiles>NCC(=O)NCC(=O)NCC(=O)NC(CO)C(=O)N1CCCC1C(=O)NCC(=O)NC(CO)C(=O)NC(CCCCNC(=O)CC(=O)O)C(=O)O</smiles>

$\mathrm{NH}_{2}$-GGG-S(PGS) ${ }_{4}$-K( $(\varepsilon-\mathrm{NH}-\mathrm{C} 18$-diacid)-OH (FA4)

Fig. 2 Structures of four FA motifs (FA1-FA4) used for the preparation of Fab bioconjugates. FA, fatty acid. 


\section{Enzyme-Linked Immunosorbent Assay for Measuring Antigen- and Albumin-Binding Activities}

In this study, TNF- $\alpha$ (antigen) binding activities and HSA (albumin) binding activities of Fab bioconjugates were assessed using recombinant human TNF- $\alpha$ (GenScript Inc., China) and HSA (Sigma-Aldrich, United States) binding ELISA methods, respectively. ELISA plates (96-well, MaxiSorp F96, Nunc) coated with $100 \mathrm{ng} /$ well TNF- $\alpha$ and 96-well plates (Sangon Biotech, China) coated with $2 \mu \mathrm{g} /$ well HSA were used for antigen- and albumin-binding assay, respectively. After coating, the plates were blocked with dry milk in PBS and incubated with serially diluted Fab bioconjugates in PBS. Goat anti-human immunoglobulin $\mathrm{G}$ (Fab specific)-peroxidase conjugate in PBS was then incubated in each well. Following $2 \mathrm{~mol} / \mathrm{L} \mathrm{H}_{2} \mathrm{SO}_{4}$ stop, the absorbance at $450 \mathrm{~nm}$ was measured using SpectraMax I3X Microplate reader (Molecular Devices Company, United States). For convenient comparison, the maximal optical density (OD) value of the each Fab bioconjugate was set to $100 \%$ in TNF- $\alpha$ binding ELISA.

\section{Cell Culture and Treatment}

L929 cells were cultured in Minimum Essential Medium (MEM; Gibco/Thermo Fisher Scientific, United States) containing $10 \%$ fetal bovine serum (FBS; Thermo Fisher Scientific, United States) at $37^{\circ} \mathrm{C}$ in a $5 \% \mathrm{CO}_{2}$ humidified atmosphere.

L929 cells $(20,000$ cells/well) were seeded in 96-well plates (Corning, United States) and then treated with different concentrations of Fab-FA3 and adalimumab Fab analog (diluted in MEM supplemented with $2 \% \mathrm{FBS}$ and $250 \mu \mathrm{g} / \mathrm{mL}$ HSA). Then, $10 \mathrm{ng} / \mathrm{mL}$ of TNF- $\alpha$ and $1 \mu \mathrm{g} / \mathrm{mL}$ of actinomycin D were added into the serial dilutions of Fab-FA3 and Fab analog. The mixtures were incubated with $\mathrm{L} 929$ cells for 17 hours. Cell viability and the TNF- $\alpha$-neutralizing activity were determined at $450 \mathrm{~nm}$ absorbance.

\section{Pharmacokinetic Studies in Mice}

The use of mice was approved by the Animal Care Committee of Chia Tai Tianqing Pharmaceutical Group. C57BL/6 mice (female, weight between 18 and $20 \mathrm{~g}$ ) were randomly divided into two groups: Fab-FA3 and Fab analog. Mice in Fab-FA3 and Fab analog groups were administered with $5 \mathrm{mg} / \mathrm{kg}$ of Fab-FA3 and adalimumab Fab analog, respectively. Blood samples were collected at 10 minutes, 30 minutes, 1 hour, 2 hours, 4 hours, 8 hours, 12 hours, 24 hours, 36 hours, and 72 hours by retro-orbital bleeding $(n=5$ mice per timepoint). The blood samples were centrifuged at $1,200 \times \mathrm{g}$ for 10 minutes at $4^{\circ} \mathrm{C}$. The plasma was stored at $-80^{\circ} \mathrm{C}$ before further analysis.

The concentrations of Fab-FA3 and adalimumab Fab ana$\log$ in plasma samples were quantified using TNF- $\alpha$-binding ELISA (as described above). The pharmacokinetic parameters, such as elimination half-life $\left(t_{1 / 2} \beta\right)$, volume of distribution, clearance $(\mathrm{CL})$, and area under the concentrationtime curve (AUC), were calculated based on a two-compartment model using DAS software version 2.0 (Mathematical Pharmacology Professional Committee of China, China).

\section{Results}

\section{Preparation and Identification of Fab Analog and FA Motifs}

The adalimumab Fab analog was expressed in E. coli periplasmic space, extracted into the periplasmic fraction, purified by affinity chromatography, and analyzed using SDSPAGE and UPLC-QTOF-MS (ultra-performance liquid chromatography to quadrupole time-of-flight mass spectrometry). As shown in -Fig. 3, Fab analog existed in the periplasmic fraction in a soluble form with a high expression level (lane 1) and was highly pure in elution fraction (lane 3). Meanwhile, under reducing conditions, the reduced heavy and light chains were visible (lane 4), confirming the disulfide bond formation in the Fab analog. Additionally, UPLCQTOF-MS showed that the observed mass of Fab analog was 49,522.18 Da, which was in accordance with its theoretical mass (49,523.12 Da), indicating its integrity (-Table 1$)$.

To prepare Fab bioconjugates, 4 FA motifs (FA1, FA2, FA3, and FA4) were designed and synthesized (-Fig. 2). The FA motifs were synthesized by a solid-phase synthesizer according to Fmoc chemistry and purified by reverse-phase chromatography. The MS data for highly purified FA motifs are shown in - Table 1. The observed molecular weights for FA1 $\quad\left(\mathrm{NH}_{2} \text {-GGG-(AEEA) }\right)_{2}$-K( $(\varepsilon-\mathrm{NH} \text {-(AEEA) })_{2}$-E-C18-diacid)$\mathrm{OH})$, FA2 ( $\mathrm{NH}_{2}$-GGG-(AEEA) $)_{2}-\mathrm{K}(\varepsilon-\mathrm{NH}-\mathrm{C} 18$-diacid)-OH), FA3 $\left(\mathrm{NH}_{2}-\mathrm{GGG}-\mathrm{A}(\mathrm{PA})_{6}-\mathrm{K}(\varepsilon-\mathrm{NH}-\mathrm{C} 18-\mathrm{diacid})-\mathrm{OH}\right)$, and FA4 $\left(\mathrm{NH}_{2}-\right.$

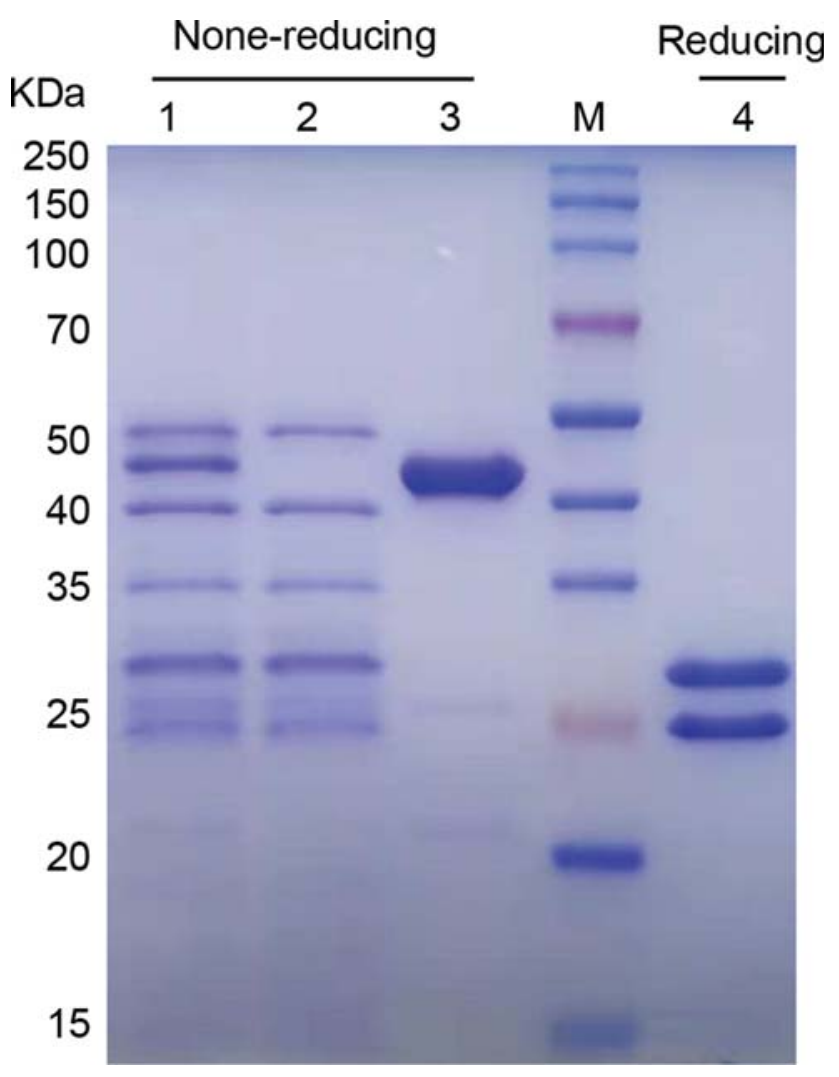

Fig. 3 SDS-PAGE analysis of the Fab analog. Lane 1: periplasmic fraction; lane 2: flow-through of protein L affinity chromatography; lanes 3 and 4: the elution fractions from protein L affinity chromatography; lane M: molecular weight markers. SDS-PAGE, sodium dodecyl sulfate polyacrylamide gel electrophoresis. 
e164 Extending the Half-Life of Adalimumab Fab Zhang et al.

Table 1 MS data (Da) for Fab analog, FA motifs, and Fab bioconjugates

\begin{tabular}{|l|l|l|l|l|l|}
\hline $\begin{array}{l}\text { Fatty acid } \\
\text { motif }\end{array}$ & $\begin{array}{l}\text { Theoretical } \\
\text { MS }\end{array}$ & $\begin{array}{l}\text { Observed } \\
\text { MS }\end{array}$ & Fab and bioconjugate & $\begin{array}{l}\text { Theoretical } \\
\text { MS }\end{array}$ & $\begin{array}{l}\text { Observed } \\
\text { MS }\end{array}$ \\
\hline & & & Fab analog & $49,523.12$ & $49,522.18$ \\
\hline FA1 & $1,322.25$ & $1,322.93$ & Fab-FA1 & $49,891.08$ & $49,891.73$ \\
\hline FA2 & 902.80 & 903.64 & Fab-FA2 & $49,471.79$ & $49,471.74$ \\
\hline FA3 & $1,692.64$ & $1,693.76$ & Fab-FA3 & $50,261.91$ & $50,262.05$ \\
\hline FA4 & $1,664.44$ & $1,665.64$ & Fab-FA4 & $50,233.79$ & $50,233.70$ \\
\hline
\end{tabular}

Abbreviations: FA, fatty acid; Fab, antigen-binding fragment; MS, mass spectrometry.

GGG-S(PGS $)_{4}-\mathrm{K}(\varepsilon-\mathrm{NH}-\mathrm{C} 18-$ diacid $\left.)-\mathrm{OH}\right) \quad$ were $\quad 1,322.93$, 903.64, 1,693.76, and 1,665.64 Da, respectively, all in accordance with the corresponding theoretical molecular weights.

\section{Successful Generation of Fab Bioconjugates through SrtA-Mediated Conjugation between Fab Analog and FA Motif}

It has been confirmed that the half-lives of proteins and peptides in circulation will be enhanced through their conjugation with FAs via the noncovalent association with albumin. ${ }^{13}$ Given the widespread use of SrtA for covalent protein modification, ${ }^{15-17}$ we further prepared Fab bioconjugates using SrtA and FA motifs. As shown in - Fig. 4, in the process of SrtA-mediated transpeptidation, the cysteine-184 (Cys184) of SrtA specifically attaches to the carbonyl carbon of $\mathrm{T}$ in LPETGG, leading to the ejection of the C-terminal fragment and the simultaneous formation of an acyl-enzyme intermediate, which further reacted with a FA motif comprising $\mathrm{N}$-terminal glycine to establish a new peptide bond between the threonine residue and glycine residue, thereby generating the Fab bioconjugate. ${ }^{16,25}$

Fab-FA1 was initially produced by the conjugation of adalimumab Fab analog with FA1 using SrtA. SDS-PAGE analysis ( - Fig. 5A) showed a high proportion of Fab-FA1 in the reaction mixture (lane 1 ), verifying the successful establishment of SrtA-mediated FA modification. Fab-FA1 with high purity was additionally obtained by $\mathrm{Ni}^{2+}$ affinity chromatography, which exhibited slightly larger molecular weight than the unreacted Fab analog (lane 2), further confirming the result.

All Fab-FA2, Fab-FA3, and Fab-FA4 were prepared in a similar manner, and migrated as single electrophoretic bands (-Fig. 5B), with an increase in the molecular weight due to the modified FA motifs. Furthermore, each of the FabFA bioconjugates was examined via UPLC-QTOF-MS, which showed correct molecular weights (-Table $\mathbf{1}$ ).

\section{Determining Antigen-Binding Activities of the Fab Bioconjugates}

ELISA assay was conducted to evaluate the TNF- $\alpha$-binding activities of Fab-FAs. As shown in - Fig. 6A, both Fab bioconjugates (Fab-FA1, Fab-FA2, Fab-FA3, and Fab-FA4) and Fab analog effectively bound TNF- $\alpha$. The TNF- $\alpha$-binding activities ( $\mathrm{EC}_{50}$ values) of the Fab bioconjugates ranged from 1.31 to $2 \mathrm{nmol} / \mathrm{L}$, which were not significantly different from that of the Fab analog $\left(\mathrm{EC}_{50}=1.37 \mathrm{nmol} / \mathrm{L}\right)$.

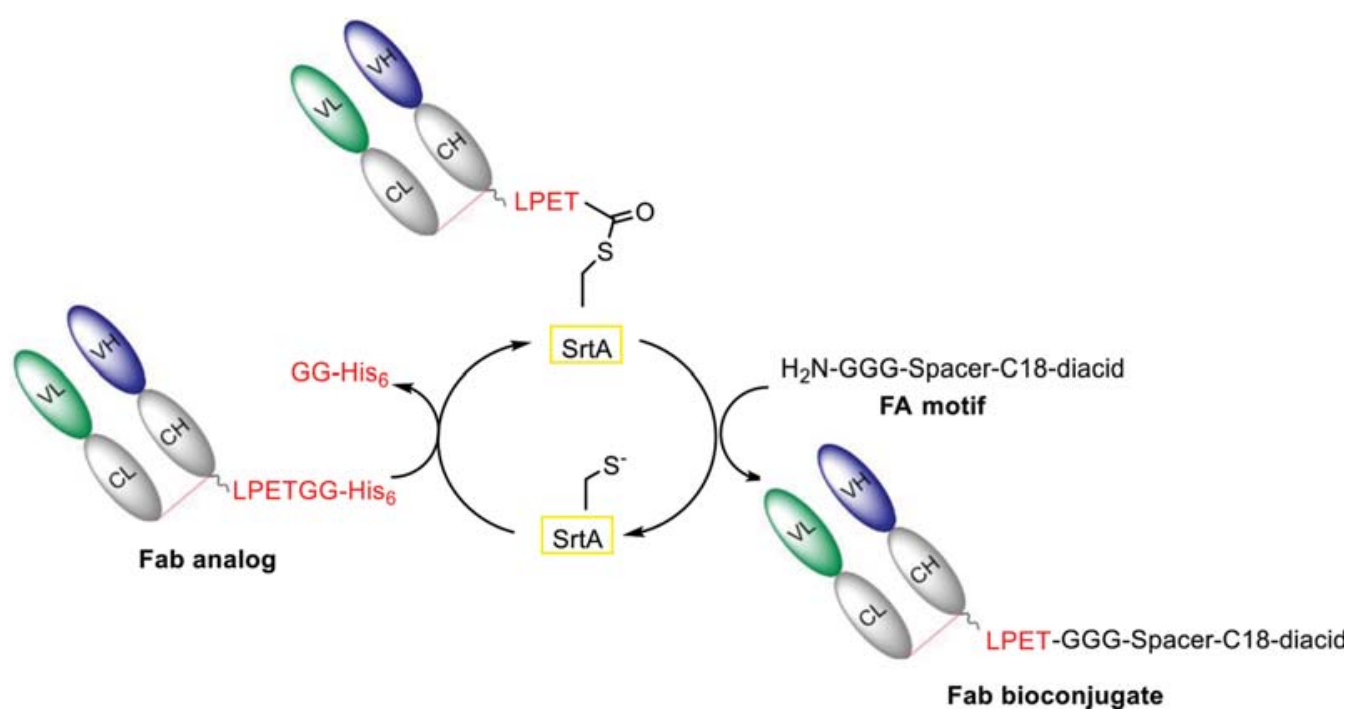

Fig. 4 Schematic diagram of the SrtA-mediated FA modification where SrtA attaches to the carbonyl carbon of the Thr residue (T-G) and appends a FA motif. FA, fatty acid. 
A

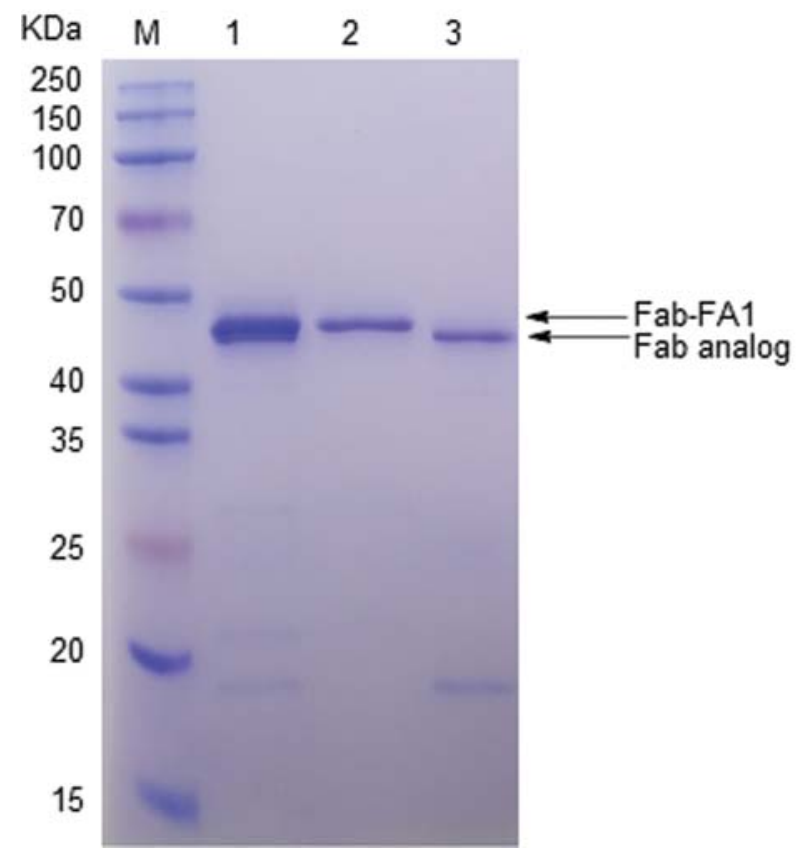

B

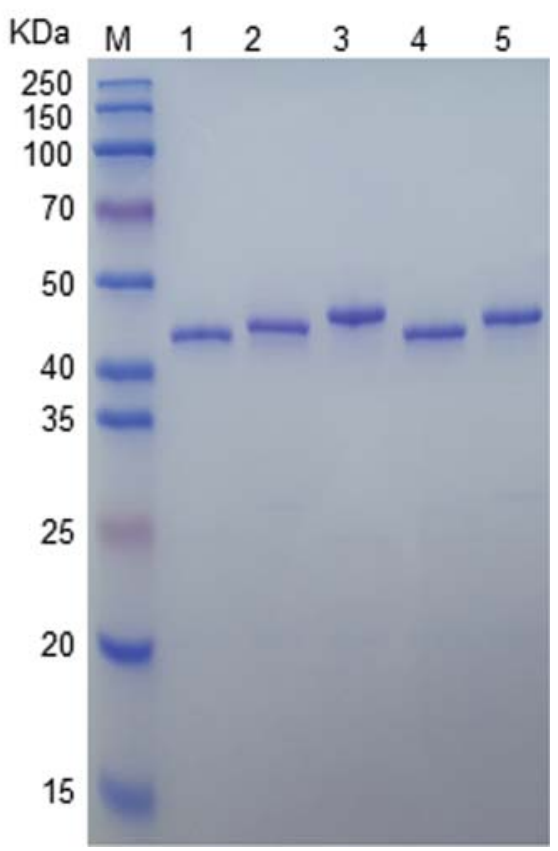

Fig. 5 SDS-PAGE analysis of the Fab bioconjugates. (A) Purification of Fab-FA1 bioconjugate via $\mathrm{Ni}^{2+}$ affinity chromatography. Lane 1: reaction mixture; lane 2: flow-through fraction; lane 3: elution fraction; lane M: molecular weight markers. (B) Identification of purified Fab bioconjugates. Lanes 1-5 represent Fab analog, Fab-FA4, Fab-FA3, Fab-FA2, and Fab-FA1, respectively. Lane M represents molecular weight markers. SDS-PAGE, sodium dodecyl sulfate polyacrylamide gel electrophoresis.

A

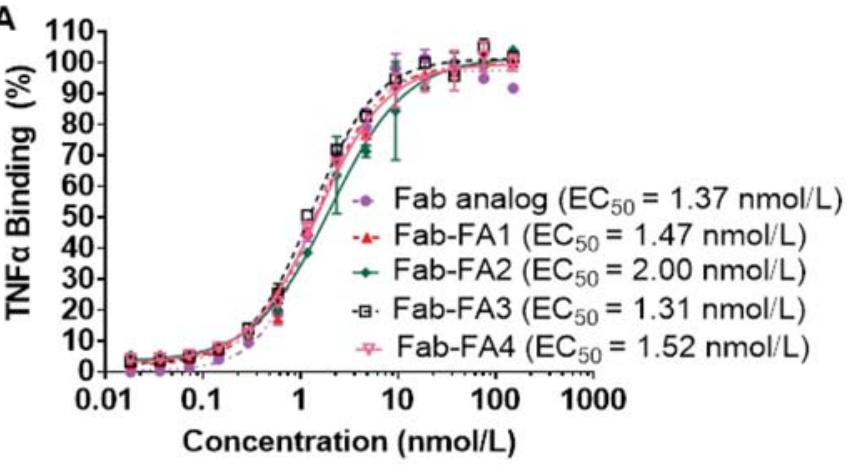

C

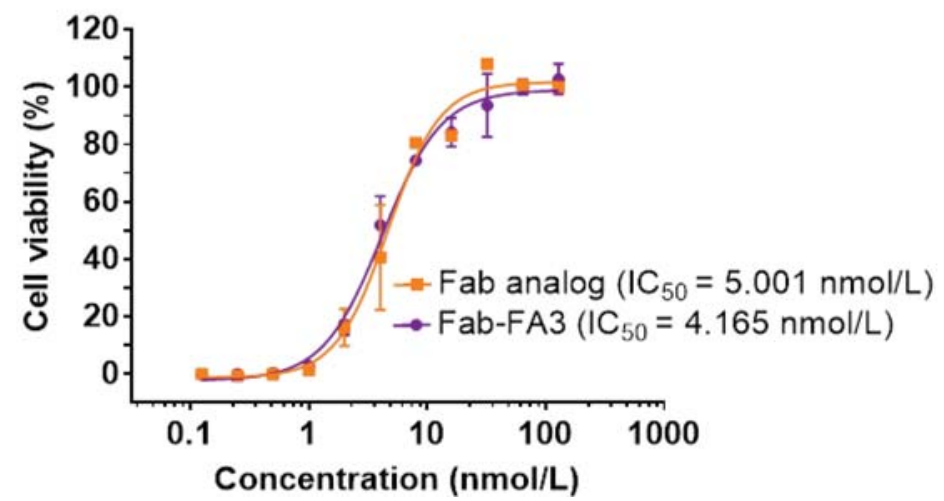

B
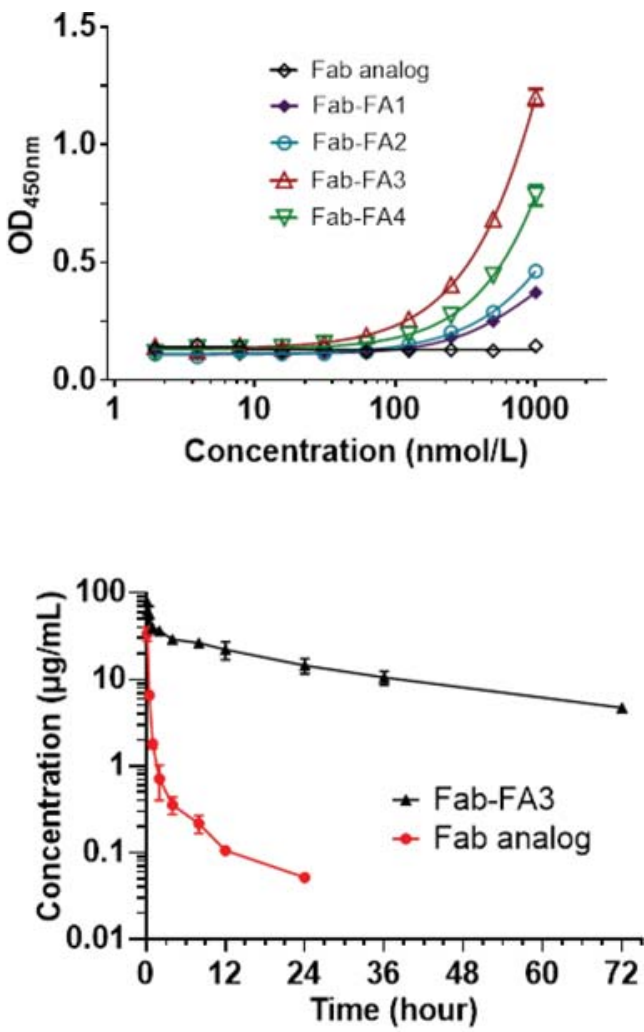

Fig. 6 Evaluation of Fab bioconjugates for (A) TNF- $\alpha$ binding by ELISA assay; (B) HSA binding by ELISA assay; (C) anti-TNF- $\alpha$ activity using L929 cells by the CCK- 8 method; and (D) the serum concentration of Fab-FA3 and Fab analog in mice using TNF- $\alpha$-binding ELISA. ELISA, enzyme-linked immunosorbent assay; HSA, human serum albumin; TNF, tumor necrosis factor. 


\section{Determining Albumin-Binding Activities of the Fab Bioconjugates}

ELISA assay was conducted to evaluate the albumin-binding activities of Fab-FAs. As shown in - Fig. 6B, the OD value of Fab analog did not change significantly; however, the OD value of Fab-FAs increased in a dose-depended manner with the maximum effect being observed with Fab-FA3. At a uniformed concentration of $1,000 \mathrm{nmol} / \mathrm{L}$, for example, the highest signal was observed for Fab-FA3, followed by FabFA4, Fab-FA2, and Fab-FA1. Therefore, the relative rank affinity for HSA was measured as Fab-FA3 $>$ Fab-FA4 $>$ Fab-FA2 $>$ Fab-FA1. Thus, Fab-FA3 was selected as the lead candidate for further investigation.

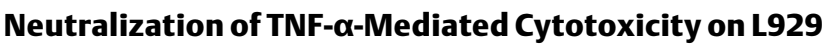 Cells}

The CCK-8 method was used to assess the effect of Fab-FA3/ Fab analog on TNF- $\alpha$-mediated cytotoxicity on L929 cells. - Fig. $6 \mathrm{C}$ shows that the $\mathrm{IC}_{50}$ for Fab-FA3 was 4.165 $\mathrm{nmol} / \mathrm{L}$, while the $\mathrm{IC}_{50}$ for Fab analog was $5.001 \mathrm{nmol} / \mathrm{L}$, demonstrating a similar TNF- $\alpha$ inhibition in the two groups in the presence of albumin. Our data suggest that the presence of albumin or binding of Fab-FA3 to albumin did not interfere with TNF- $\alpha$ binding.

\section{Pharmacokinetics of Fab-FA3 and Adalimumab Fab Analog}

Plasma concentration-time profiles of Fab-FA3 and Fab analog were assessed using TNF- $\alpha$-binding ELISA to determine their in vivo half-lives in mice. - Fig. 6D shows that the concentration of Fab analog in mouse plasma was undetectable after 24 hours, demonstrating the rapid elimination of Fab in vivo. However, the concentration of Fab-FA3 at 72 hours remains detectable (at $\sim 5 \mu \mathrm{g} / \mathrm{mL}$ ), confirming the prolonged retention time of Fab-FA3 in vivo. As shown in -Table 2, the clearance rate of Fab-FA3 was only $5.3 \pm 0.8\left(\mathrm{~mL}[\mathrm{~h} \mathrm{~kg}]^{-1}\right)$, which was 33.5 -fold lower than that of the Fab analog $\left(177.7 \pm 24.1, \mathrm{~mL}[\mathrm{~h} \mathrm{~kg}]^{-1}\right)$. Therefore, Fab-FA3 exhibited a 15.2-fold longer $t_{1 / 2} \beta(19.86 \pm 3.25$ hours) when compared with that $(1.31 \pm 0.38$ hours $)$ of the Fab analog. These results demonstrate the improvement of the pharmacokinetic properties of Fab-FA3 and the successful establishment of SrtA-mediated FA modification for prolonging the half-life of adalimumab Fab in vivo.

Table 2 Pharmacokinetic properties of Fab-FA3 and Fab analog

\begin{tabular}{|l|l|l|}
\hline & Fab-FA3 & Fab analog \\
\hline$t_{1 / 2} \beta(\mathrm{h})$ & $19.86 \pm 3.25$ & $1.31 \pm 0.38$ \\
\hline $\mathrm{Vd}(\mathrm{mL} \mathrm{kg}$ & $59.2 \pm 16.5$ \\
\hline $\mathrm{CL}\left(\mathrm{mL}[\mathrm{h} \mathrm{kg}]^{-1}\right)$ & $52.3 \pm 14.4$ & $177.7 \pm 24.1$ \\
\hline $\mathrm{AUC}_{(0-\infty)}\left(\left[\mathrm{mg} \mathrm{L}^{-1}\right] \mathrm{h}\right)$ & $1,114.95 \pm 129.48$ & $28.59 \pm 3.62$ \\
\hline
\end{tabular}

Abbreviations: AUC, area under the concentration-time curve; $\mathrm{CL}$, clearance; Fab, antigen-binding fragment; $t_{1 / 2} \beta$, elimination half-life; $\mathrm{Vd}$, volume of distribution.

\section{Discussion}

Adalimumab, a human recombinant anti-TNF- $\alpha$ monoclonal antibody, is the world's best-selling drug for treatment of TNF$\alpha$-mediated inflammatory diseases with favorable therapeutic effect. ${ }^{3}$ However, the use of adalimumab may be limited among some patients due to its the high production cost, the restricted tissue distribution, and the undesired immunoreactions., ${ }^{4,5}$ Fab, derived from adalimumab, retains the antigen-binding activity and can compensate for the above-mentioned imperfections. However, Fab unsatisfactorily exhibits poor pharmacokinetics due to its small size, which limits its therapeutic applications. ${ }^{5}$ Thus, the successful construction of long-acting adalimumab Fab may represent an alternative for adalimumab in treating TNF- $\alpha$-associated diseases.

This study is the first to describe the SrtA-mediated conjugation of Fab with a modified FA. We constructed Fab analog by linking the recognition sequence of SrtA (LPETGG) and $\mathrm{His}_{6}$ tag to the heavy-chain C-terminus of Fab via $\left(\mathrm{G}_{4} \mathrm{~S}\right)_{3}$ (-Fig. 1). The flexible linker, $\left(\mathrm{G}_{4} \mathrm{~S}\right)_{3}$, was designed to enhance the solubility, folding, and stability of its fusion protein. ${ }^{22}$ Then, FA motifs with different linkers were synthesized (-Fig. 2) and conjugated to the adalimumab Fab analog through SrtA (-Fig. 4). Our data further confirmed the similar TNF- $\alpha$ binding activity between Fab analog and Fab bioconjugates ( $\mathbf{- F i g . 6 A}$ ), suggesting that the linking of Fab to the FA motif does not reduce the inherent bioactivity of Fab.

In terms of albumin binding capacity of Fab-FAs, different linkers in FA motifs exerted different affinities for HSA with the maximal effect shown by Fab-FA3. We thus speculate that $\mathrm{A}(\mathrm{PA})_{6}$ in $\mathrm{FA3}$ is a superior linker or spacer when compared with (AEEA) $)_{2}$ in FA2 and $\mathrm{S}(\mathrm{PGS})_{4}$ in FA4, and this may be due to its rigid structure and relatively weaker hydrophilic property. AEEA-based linker has been widely used as a hydrophilic spacer in the synthesis of FA motifs, ${ }^{21,26}$ however, our data showed that the albuminbinding activity of Fab-FA1 is smaller to Fab-FA2, demonstrating that the increased hydrophilic nature might weaken the hydrophobic interaction between FA and albumin.

We further assessed the TNF- $\alpha$-neutralizing efficiency and the pharmacokinetics of Fab-FA3. Our data confirmed the similar inhibition effect of Fab-FA3 and Fab analog on TNF- $\alpha$ mediated cytotoxicity on L929 cells (-Fig. 6C), indicating that Fab-FA3 preserved the in vivo antigen-blocking activity of Fab analog even if it coupled with endogenous albumin. For pharmacokinetics, our data displayed a sustained plasma presence of Fab-FA3 with a 15.2-fold increase in $t_{1 / 2} \beta$ (19.86 hours) and a 33.5 -fold decrease in CL (5.3 mL $[\mathrm{h} \mathrm{kg}]^{-1}$ ) when compared with its control. Our study suggests the successful generation of Fab bioconjugate with prolonged half-life and anti-TNF- $\alpha$ activity.

\section{Conclusion}

An effective approach was established for the development of Fab bioconjugate showing sustained activity through SrtAmediated conjugation of Fab analog with a FA motif. Fab-FA3 with anti-TNF- $\alpha$ activity exhibited satisfying 
pharmacokinetics in vivo, and may represent an alternative drug candidate for adalimumab in treating TNF- $\alpha$-mediated inflammatory disease.

\section{Conflicts of Interest}

The authors declare no conflict of interest.

\section{References}

1 Baud V, Karin M. Signal transduction by tumor necrosis factor and its relatives. Trends Cell Biol 2001;11(09):372-377

2 Mitoma H, Horiuchi T, Tsukamoto H, Ueda N. Molecular mechanisms of action of anti-TNF- $\alpha$ agents - comparison among therapeutic TNF- $\alpha$ antagonists. Cytokine 2018;101:56-63

3 Steeland S, Libert C, Vandenbroucke RE. A new venue of TNF targeting. Int J Mol Sci 2018;19(05):1442

4 Hansel TT, Kropshofer H, Singer T, Mitchell JA, George AJ. The safety and side effects of monoclonal antibodies. Nat Rev Drug Discov 2010;9(04):325-338

5 Chames P, Van Regenmortel M, Weiss E, Baty D. Therapeutic antibodies: successes, limitations and hopes for the future. $\mathrm{Br} J$ Pharmacol 2009;157(02):220-233

6 Rader C. Overview on concepts and applications of Fab antibody fragments. Curr Protoc Protein Sci 2009;Chapter 6:Unit 6.9

7 Flanagan RJ, Jones AL. Fab antibody fragments: some applications in clinical toxicology. Drug Saf 2004;27(14):1115-1133

8 Rosa J, Sabelli M, Soriano ER. Prefilled certolizumab pegol (Cimzia (®)) syringes for self-use in the treatment of rheumatoid arthritis. Med Devices (Auckl) 2010;3:25-31

9 Schlapschy M, Binder U, Börger C, et al. PASylation: a biological alternative to PEGylation for extending the plasma half-life of pharmaceutically active proteins. Protein Eng Des Sel 2013;26 (08):489-501

10 Nguyen A, Reyes AE II, Zhang M, et al. The pharmacokinetics of an albumin-binding Fab (AB.Fab) can be modulated as a function of affinity for albumin. Protein Eng Des Sel 2006;19(07):291-297

11 Schlapschy M, Theobald I, Mack H, Schottelius M, Wester HJ, Skerra A. Fusion of a recombinant antibody fragment with a homo-amino-acid polymer: effects on biophysical properties and prolonged plasma half-life. Protein Eng Des Sel 2007;20 (06):273-284

12 van Witteloostuijn SB, Pedersen SL, Jensen KJ. Half-life extension of biopharmaceuticals using chemical methods: alternatives to PEGylation. ChemMedChem 2016;11(22):2474-2495
13 Zorzi A, Linciano S, Angelini A. Non-covalent albumin-binding ligands for extending the circulating half-life of small biotherapeutics. MedChemComm 2019;10(07):1068-1081

14 Bech EM, Pedersen SL, Jensen KJ. Chemical strategies for half-life extension of biopharmaceuticals: lipidation and its alternatives. ACS Med Chem Lett 2018;9(07):577-580

15 Ilangovan U, Ton-That H, Iwahara J, Schneewind O, Clubb RT. Structure of sortase, the transpeptidase that anchors proteins to the cell wall of Staphylococcus aureus. Proc Natl Acad Sci U S A 2001;98(11):6056-6061

16 Lieser RM, Yur D, Sullivan MO, Chen W. Site-specific bioconjugation approaches for enhanced delivery of protein therapeutics and protein drug carriers. Bioconjug Chem 2020;31(10): 2272-2282

17 Levary DA, Parthasarathy R, Boder ET, Ackerman ME. Proteinprotein fusion catalyzed by sortase A. PLoS One 2011;6(04): e18342

18 Popplewell AG, Sehdev M, Spitali M, Weir AN. Expression of antibody fragments by periplasmic secretion in Escherichia coli. Methods Mol Biol 2005;308:17-30

19 Newton JM, Vlahopoulou J, Zhou Y. Investigating and modelling the effects of cell lysis on the rheological properties of fermentation broths. Biochem Eng J 2017;121:38-48

20 Li Q, Aucamp JP, Tang A, Chatel A, Hoare M. Use of focused acoustics for cell disruption to provide ultra scale-down insights of microbial homogenization and its bioprocess impact-recovery of antibody fragments from rec E. coli. Biotechnol Bioeng 2012; 109(08):2059-2069

21 Lau J, Bloch P, Schäffer L, et al. Discovery of the once-weekly glucagon-like peptide-1 (GLP-1) analogue semaglutide. J Med Chem 2015;58(18):7370-7380

22 Chen X, Zaro JL, Shen WC. Fusion protein linkers: property, design and functionality. Adv Drug Deliv Rev 2013;65(10):1357-1369

23 Gosselin ML, Fabrizio D, Swain JF, et al. Serum albumin binding molecules. US Patent 8969289. March, 2015

24 Zhang QB, Huang ZQ, Lu JG, Zhao WJ, Feng J. Prokaryotic expression, purification and activity determination of Sortase A enzyme mutant [in Chinese]. Carol J Pharm 2020;51(01):37-47

25 Pishesha N, Ingram JR, Ploegh HL. Sortase A: a model for transpeptidation and its biological applications. Annu Rev Cell Dev Biol 2018;34:163-188

26 Lau J, Hansen TK, Madsen K, et al. Novel GLP-1 derivatives. WO Patent 2005027978. March, 2005 